Ebisu Ebisu

Études japonaises Études japonaises

$51 \mid 2014$

Le rapprochement franco-japonais dans l'entre-deuxguerres

\title{
Une leçon japonaise : la gravure sur bois en
} couleurs en France, 1889-1939

1889 １939 年のフランスの木版表現にみられる浮世絵の影響、そし て出版

The Colour Woodcut in France Using Japanese Techniques (1889-1939)

\section{Philippe Le Stum}

\section{OpenEdition}

Journals

Édition électronique

URL : http://journals.openedition.org/ebisu/1484

DOI : 10.4000/ebisu. 1484

ISSN : 2189-1893

Éditeur :

Institut français de recherche sur le Japon (UMIFRE 19 MAEE-CNRS), Maison franco-japonaise

Référence électronique

Philippe Le Stum, « Une leçon japonaise : la gravure sur bois en couleurs en France, 1889-1939», Ebisu [En ligne], 51 | 2014, mis en ligne le 01 novembre 2014, consulté le 01 mai 2019. URL : http:// journals.openedition.org/ebisu/1484; DOI : 10.4000/ebisu.1484 


\title{
Une leçon japonaise : la gravure sur bois en couleurs en France, 1889-1939
}

\author{
Philippe LE STUM*
}

On connaît aujourd'hui parfaitement l'impact de la révélation des estampes japonaises sur la peinture occidentale à partir des années 1860. Plusieurs expositions et publications ont été consacrées à ce phénomène ${ }^{1}$. Elles ont en particulier mis en évidence l'influence de l'estampe japonaise sur les peintres français, en matière de sujet, de composition et d'utilisation de la couleur. On a aussi, parfois, relevé l'influence du Japon sur des graveurs en taille-douce, c'est-à-dire des graveurs sur cuivre français, tels que Henri Guérard ${ }^{2}$.

Ce dont nous traitons ici est un épisode beaucoup moins connu de l'histoire du japonisme. À l'exception d'Henri Rivière, la plupart des artistes que nous évoquerons sont en effet tombés dans l'oubli. Quelques-uns font actuellement l'objet, en France, d'une redécouverte tardive ${ }^{3}$. Jusqu'à présent les historiens d'art ont essentiellement été sensibles aux aspects esthétiques et formels du japonisme. Dans le courant japoniste que nous étudions,

* Docteur en histoire de l'art, directeur du Musée départemental breton, Quimper.

1. Siegfried Wichmann, Japonism. The Japanese influence on Western Art in the $19^{\text {th }}$ and $20^{\text {th }}$ centuries, New York, Park Lane, 1985 et Japonisme, cat. expo., Paris, Galeries nationales du Grand Palais, Réunion des musées nationaux, 1988.

2. Paris, 28 avril $1846-25$ mars 1897.

3. Nous leur avons consacré une exposition, accompagnée d'un catalogue, en 2012 : Ph. Le Stum (dir.), Japon, Paris, Bretagne, 1880-1930. La gravure sur bois en couleurs, cat. expo., Quimper, Musée départemental breton, 2012. 
la technique fut plus essentielle encore que l'esthétique, puisqu'il s'agit de l'expérimentation et de l'adoption, par des graveurs sur bois français, des techniques japonaises de gravure et d'impression en couleurs. Ce mouvement, qui concerna d'abord l'exécution d'estampes, eut des conséquences importantes sur l'évolution du livre illustré.

\section{Naissance de la gravure sur bois originale}

Le phénomène ne débuta qu’à la fin des années 1880, soit plus de vingt ans après les premiers effets de la découverte de l'estampe japonaise sur la peinture et la gravure sur cuivre françaises. Ce décalage chronologique vient du fait que la curiosité des graveurs français pour les procédés techniques de la gravure japonaise fut conditionnée par le changement de statut artistique et critique de la gravure sur bois en France. Or, cette évolution ne survint que dans le courant de la décennie 1880 .

Jusqu'à cette époque, la gravure sur bois n'était pas considérée en France et en Occident comme un art, mais comme une technique artisanale de reproduction ${ }^{4}$. La gravure sur bois imprimée en couleurs était ignorée. En revanche, la gravure sur bois en noir était très abondamment utilisée dans l'illustration des revues ou des livres. Elle avait en effet l'avantage, par rapport aux autres formes de gravure, de pouvoir être imprimée en même temps que les caractères typographiques. Mais quelle que soit leur qualité, ces gravures n'étaient que des illustrations auxquelles on n'accordait pas le statut d'œuvre d'art. Elles étaient exécutées par des artisans graveurs professionnels, dont le métier consistait à graver dans le bois un dessin effectué par un autre intervenant, un artiste comme par exemple Gustave Doré. Les artistes dessinaient sur la matrice ; celle-ci était ensuite gravée par des graveurs de métier. Ces derniers travaillaient souvent en équipe, comme le montre une illustration gravée par Tony Beltrand en 1897, pour la revue L'Image (fig. 1)

On peut remarquer que la division du travail qui s'était instaurée en Occident, entre l'artiste dessinateur, puis les artisans graveurs et imprimeurs, était globalement identique au processus d'exécution des estampes

4. Sur cet aspect, voir : Remi Blachon, La gravure sur bois au XIXe siècle. L'âge du bois debout, Paris, Les éditions de l'Amateur, 2001. 


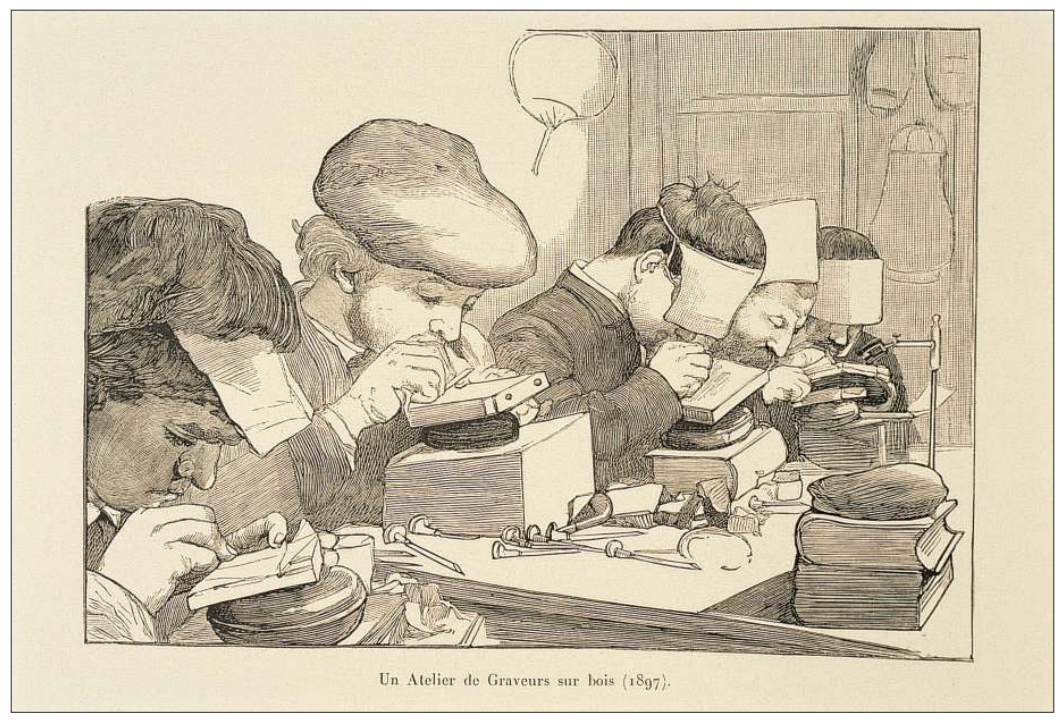

\section{Fig. 1.}

Tony Beltrand, Un atelier de graveurs sur bois.

Gravure sur bois publiée dans L'Image, sept. 1897, p. 316, H. $9,8 \mathrm{~cm} ;$ L. 14,2 cm. Coll. Musée départemental breton (Conseil général du Finistère), Quimper.

dans les ateliers japonais. Pourtant, paradoxalement, ce fut d'abord sur le rejet de cette division du travail que s'opéra l'adoption des procédés japonais par des graveurs français.

La manifestation essentielle du renouveau de la gravure sur bois en France fut en effet l'avènement de ce que l'on appelé la gravure sur bois " originale ». C'est-à-dire celle pour l'exécution de laquelle l'artiste gravait lui-même au lieu de confier son dessin à un graveur professionnel. La gravure sur bois changea ainsi de statut : de simple procédé de reproduction, elle devenait une forme d'art à part entière. La gravure sur bois allait donc sortir du livre ou de la revue et pouvoir être collectionnée, mais aussi accrochée sur le mur du logis ou d'une salle d'exposition. Pour mieux répondre à cette nouvelle fonction décorative, quelques graveurs sur bois résolurent d'acquérir les techniques de l'impression en couleurs. 


\section{La découverte des techniques japonaises de la gravure sur bois en couleurs}

La découverte, la vogue et l'étude de l'estampe japonaise en France furent décisives dans cette évolution. L'estampe japonaise faisait l'objet d'expositions, était diffusée par des galeries spécialisées ou présentée dans des revues telles que Le Japon artistique, du galeriste Siegfried Bing 5 . Cette découverte conduisit la critique et les collectionneurs à porter un nouveau regard sur la gravure sur bois. Cette technique fut désormais reconnue comme capable de produire des œuvres d'art. Soucieux d'être à leur tour considérés comme des artistes, quelques graveurs français résolurent de démontrer qu'ils pouvaient créer des estampes qui, à l'égal des estampes japonaises, seraient reconnues comme des œuvres d'art de valeur.

Ces graveurs savaient graver le bois. En revanche, ils ignoraient les techniques d'encrage et d'impression en couleurs. Ce fut donc presque uniquement par l'étude des estampes japonaises qu'ils acquirent la connaissance de ces techniques. Les trois aspects qu'ils durent découvrir et maîtriser furent :

$1^{\circ}$ - Tout d'abord, la taille d'une matrice par couleur et le système de repérage qui devait permettre de procéder à l'impression successive des différentes couleurs.

$2^{\circ}$ - Le second aspect portait sur la nature des encres utilisées. La gravure occidentale utilisait en effet des encres grasses, huileuses et assez opaques, appliquées au rouleau ${ }^{6}$. L'imprimeur japonais, lui, utilisait des pigments minéraux ou végétaux dilués dans de l'eau. La nature du produit utilisé et la manière de le poser lui permettaient ces effets de transparence et de dégradés qui séduisaient les artistes et les collectionneurs occidentaux. C'était là le point le plus fondamental. Nos graveurs s'efforcèrent de réussir à « imprimer à l'eau ».

5. Dit aussi Samuel Bing, Hambourg, 26 février 1838 - Vaucresson, 6 septembre 1905. Voir : collectif, Les origines de l'Art nouveau. La maison Bing, cat. expo. Paris, musée des Arts décoratifs, 2004.

6. Sur les techniques occidentales de gravure sur bois, voir : Morin-Jean, Manuel pratique de la gravure sur bois, Paris, Laurens, 1926, rééd. 1961. 
$3^{\circ}$ - Pour imprimer ces estampes, ils durent aussi découvrir l'usage du frotton (baren) et donc imprimer à la main sans recourir à la presse alors d'usage en Occident.

\section{Henri Rivière, le précurseur}

En France et sans doute en Europe, Henri Rivière ${ }^{7}$ fut le premier artiste à utiliser les procédés japonais pour l'exécution de gravures sur bois en couleurs. Rivière a lui-même raconté les circonstances de la réalisation de ses premiers bois imprimés à l'eau, dont l'idée lui vint d'abord de l'observation des estampes japonaises chez le galeriste Bing. Il s'en forma lui-même une belle collection selon une pratique commune à la plupart des graveurs que nous évoquerons. L'influence esthétique des estampes japonaises imprégna d'abord son travail d'illustrateur : ses dessins à la plume composés pour un conte fantastique publié en $1886^{8}$ sont parsemés d'emprunts iconographiques à Hokusai et à Hiroshige : grenouilles et crapauds à la manière du premier, pont Nihonbashi repris d'une estampe du second. Mais en 1888, Rivière résolut de comprendre et d'adopter non plus seulement le répertoire formel des estampes japonaises, mais leurs techniques de réalisation. Dans ses mémoires, que j'ai eu l'honneur de publier, il décrit ses tâtonnements et ses premières réussites. Ce texte montre parfaitement qu'à cette date, les graveurs français ne disposaient que de renseignements très incomplets sur les techniques japonaises de gravure et surtout d'impression. Ils n'avaient pas de manuels, ni encore de contacts avec des graveurs japonais, de sorte qu'ils devaient procéder par expérimentation.

« Le Japon, écrit Rivière, nous envoyait alors en vrac poteries, laques, étoffes, bronzes, objets d'art de toute sorte, des livres illustrés et surtout des estampes en couleurs qui faisaient notre enchantement. C'étaient des gravures sur bois, imprimées non pas comme chez nous avec des couleurs à l'huile, mais avec des couleurs à l'eau,

7. Paris, 11 mars 1864 - Sucy-en-Brie, 24 août 1951. Sur Rivière, voir principalement : Valérie Sueur-Hermel (dir.), Jocelyn Bouquillard, Philippe Le Stum, Catherine Meneux, Monique Moulène, Henri Rivière, entre impressionnisme et japonisme, cat. expo., Paris, Bibliothèque nationale de France, 2009.

8. Achille Mélandri, Les farfadets, conte breton, Paris, Quentin, 1886. 
tirées sans presse, à la main. Et c'est ainsi que j'avais résolu d'imprimer celles que je voulais graver. Mais par quels moyens? J'en ignorais les tours de main d'encrage, d'impression, de repérage, et je me mis à chercher. Je gravai d'abord sur du poirier en bois de fil quelques planches de trait et de couleur (de celles-ci cinq, six, sept ou huit par estampe) sans savoir comment je pourrais les imprimer. Pendant trois ou quatre mois je fis de nombreux essais et arrivai enfin au résultat désiré. C'était un peu ridicule d'inventer ainsi un procédé déjà existant. Quand je le connus plus tard, Hayashi s'amusa beaucoup de voir mes primitifs instruments qui depuis longtemps, en beaucoup mieux naturellement, servaient aux imprimeurs japonais. (...) Je fabriquais moi-même les couleurs, les broyais (...) ; j'avais heureusement pu me procurer chez un importateur plusieurs rames d'un beau papier (...) plus que centenaire, qui provenait de la liquidation d'une fabrique de Tokyo ; (..) avec un frotton de mon invention, j’imprimais mes planches à la main, à vingt exemplaires chacune ${ }^{9}$. "

Dans son atelier parisien, Rivière s'attela d'abord à la gravure de bois montrant un sujet d'actualité, le chantier de la tour Eiffel, alors en construction en prévision de l'Exposition universelle. Il laissa cependant sa série parisienne inachevée pour se consacrer à une suite composée à partir de croquis et d'aquarelles pris au cours d'étés passés en Bretagne, qui attirait alors beaucoup les artistes et l'avait séduit à son tour. Rivière consacra désormais à la Bretagne la part majeure de son œuvre, au point qu'un critique écrivit qu'il fit de cette région " une dépendance des archipels nippons ${ }^{10}$ ". Sa mầtrise de la gravure polychrome et de l'impression "à l'eau " fut révélée au public en 1892, par l'exposition de trente-trois estampes intitulées Paysages bretons, au Salon de la Société des peintres-graveurs français à la galerie Durand-Ruel ${ }^{11}$. Simultanément, l'artiste gravait les six estampes de La Mer, Études de vagues et les imprimait à l'aquarelle.

9. Henri Rivière, Les détours du chemin, souvenirs, notes et croquis (1864-1951), présenté par Ph. Le Stum, Saint-Rémy-de-Provence, Equinoxe, 2004, p. 65. Hayashi Tadamasa 林忠正 (1853-1906), auquel Rivière fait ici allusion, tenait à Paris une boutique d'estampes et d'art oriental et en organisait des expositions. Les deux hommes se lièrent d'amitié et par la suite Hayashi commanda à Rivière la décoration murale de sa maison de Tokyo. C'est notamment grâce à Hayashi que Rivière put se former une belle collection d'estampes, aujourd'hui conservée à la Bibliothèque nationale de France.

10. Claude Roger-Marx, La gravure originale au XIX siècle, Paris, Somogy, 1962, p. 237. 11. La Société des peintres-graveurs français a été fondée en 1889 à l'initiative de trois japonisants : le critique et collectionneur Philippe Burty (1830-1890) et les graveurs Félix Bracquemond (1833-1914) et Henri Guérard (1846-1897). 
À ses modèles japonais, Rivière emprunta aussi une manière de dessiner en simplifiant les formes cernées d'un trait et sans modelé. Il leur doit encore un répertoire de motifs. Par exemple, le rideau d'arbres coupés au sommet, en avant de la composition, est un motif décoratif japonais dont il tira fréquemment parti. Il a souvent été relevé que la coupure par le cadre de la composition d'un sujet situé en premier plan était un procédé que les artistes occidentaux tirèrent de leur observation des estampes japonaises. Rivière utilisa ce principe qui mène l'œil du spectateur dans la profondeur de la scène, par exemple dans le Départ des sardiniers (1893), inspiré de l'estampe Ushibori dans la province de Hitachi, l'une des Trente-six vues du mont Fuji (c. 1831-1834) par Hokusai, dont Rivière possédait une épreuve.

L'habitude adoptée par Rivière de grouper ses estampes en séries thématiques - Paysages bretons, Études de vagues, etc. - est aussi un emprunt direct aux modèles japonais. Après 1894, Rivière délaissa la gravure sur bois pour la lithographie, plus rapide d'exécution et permettant des tirages beaucoup plus nombreux. En lithographie, il édita plusieurs séries d'estampes dans lesquelles l'influence thématique et formelle du Japon est encore très présente. C'est le cas dans les études atmosphériques de la Féérie des heures (1901-1902) et bien sûr dans l'album des Trente-six vues de la tour Eiffel (1888-1902), hommage explicite aux Trente-six vues du mont Fuji de Hokusai.

\section{Auguste Lepère}

Mais revenons à la fin des années 1880 . À cette époque, la gravure en couleur des Japonais semble avoir constitué une sorte de défi technique et esthétique pour les xylographes français qui voulaient se faire reconnaître comme graveurs originaux. C'était spécialement le cas d'Auguste Lepère ${ }^{12}$, graveur professionnel et le plus réputé alors des graveurs sur bois français. En 1889 - la même année que Rivière - Lepère expérimenta lui aussi les procédés japonais de gravure et d'impression à l'eau pour l'exécution de son Palais de Justice de Paris vu du pont Notre-Dame. Il grava encore et imprima à l'eau, en 1890, un charmant sujet, une Petite Fille au pot-dit aussi On

12. Paris, 30 novembre 1849 - Domme, 20 novembre 1918. 


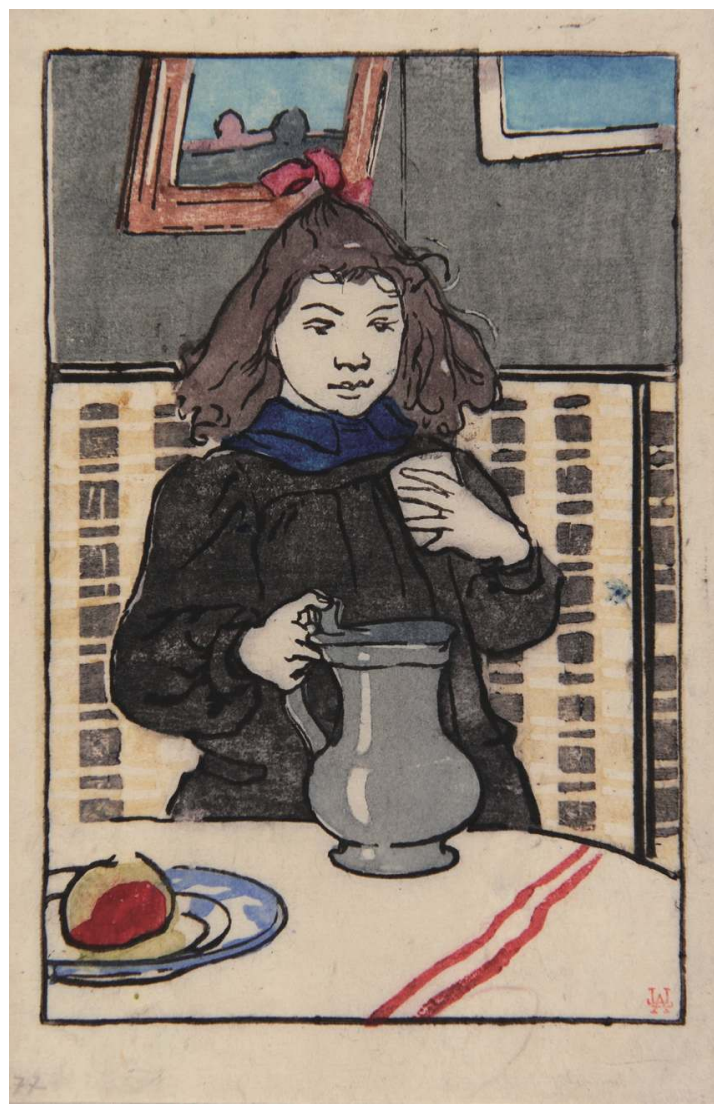

Fig. 2.

Auguste Lepère, La Petite Fille au pot ou On va goûter!

Gravure sur bois imprimée en couleurs, à l'eau, 1890.

H. $21,4 \mathrm{~cm}$; L. $14 \mathrm{~cm}$.

Coll. particulière. 
va goûter! (fig. 2) - et quelques autres estampes en couleurs, bien moins nombreuses cependant que celles de Rivière ${ }^{13}$.

Lepère était un illustrateur très recherché par les éditeurs. Il mit à profit sa maitrise de la gravure sur bois en couleurs dans plusieurs ouvrages qui comptent parmi les plus belles réalisations du livre français de la fin du XIx ${ }^{e}$ siècle. Par exemple, son Almanach pour l'année 1897 dont les saisons sont personnifiées par des parisiennes aux différents âges de la vie et dans des moments successifs de l'année ${ }^{14}$. Dans ses illustrations, gravées sur bois, du roman symboliste $\grave{A}$ rebours de Huysmans ${ }^{15}$, les emprunts iconographiques au Japon sont nombreux. La vision cauchemardesque d'un poulpe enlaçant une femme est inspirée de l'illustration de Hokusai connue en France sous le titre du Rêve de la femme du pêcheur (Tako to ama 蛸と 海女, tirée de l'album Kinoe no komatsu 喜能会之故真通, 1814). Quant à la vignette de tête du deuxième chapitre, c'est une réminiscence de la Grande Vague à Kanagawa (Kanagawa-oki nami-ura 神奈川沖浪裏) qui demeure en Occident la plus célèbre de toutes les estampes japonaises.

Il faut souligner cependant que dans le domaine de l'édition de livres les imprimeurs français n'utilisèrent pas, sauf à quelques rares exceptions, les encres diluées à l'eau, comme les Japonais, mais les encres grasses avec lesquelles ils avaient l'habitude de travailler. Ils n'employaient pas non plus le frotton (baren) ni l'impression à la main, mais la presse familière aux Occidentaux depuis le $\mathrm{Xv}^{\mathrm{e}}$ siècle. On peut donc dire que dans l'édition de livres illustrés de gravures sur bois en couleurs, le processus de gravure polychrome à plusieurs matrices était d'inspiration japonaise, tandis que les procédés d'impression demeuraient de tradition occidentale.

13. Voir : Charles Saunier, Auguste Lepère, peintre et graveur, décorateur de livres, Paris, Le Garrec, 1931.

14. Paris Almanach, texte de Charles Morice, illustrations d'Auguste Lepère, Paris, éd. Sagot, 1897.

15. Joris-Karl Huysmans, $\grave{A}$ rebours, deux cent vingt gravures sur bois en couleurs d'Auguste Lepère, Paris, Les cent bibliophiles, 1903. Nos deux exemples sont les têtes des chapitres IX et II. 


\section{Les Beltrand, père et fils}

Parmi les graveurs français pionniers de la gravure sur bois en couleurs imprimée à l'eau figure un ami et collaborateur de Lepère, Tony Beltrand ${ }^{16}$. En 1896, il fonda avec Lepère la revue L'Image, dont le but était de défendre la xylographie. En 1897, il exposa à Paris une série de dix études de femmes en costumes régionaux. Elles sont rarissimes et les tirages durent en être limités à quelques exemplaires, imprimés à la main, à l'eau. Plusieurs présentent la particularité de comporter des effets de reliefs par gaufrage, soulignant par exemple l'écume d'une vague ou indiquant la texture d'un tissu (fig. 3). Ce procédé très précieux fut inspiré à Beltrand par les surimono, estampes qui jouissaient d'un prestige particulier auprès des collectionneurs occidentaux, et dont lui-même possédait de beaux exemplaires. Tony Beltrand regrava en plus petit format ces études de femmes en costumes pour les faire imprimer - mais en noir cette fois, et à la presse et à l'encre grasse - en illustration d'un très beau livre d'inspiration Art nouveau, Croquis de Bretagne et d'ailleurs, édité en $1903^{17}$.

Tony Beltrand eut quatre fils qui furent tous graveurs professionnels. Le plus important fut Jacques Beltrand ${ }^{18}$, dont l'œuvre gravé est considérable. Sous l'influence de son père et de Lepère, il s'essaya lui aussi à la gravure en couleurs et à l'impression à l'eau. Il exécuta selon ces techniques japonaises les pages d'un Almanach contemporain de celui illustré par Lepère en 1897. Cette brochure, tirée peut-être à un ou deux exemplaires seulement, fut une des très rares tentatives françaises d'imprimer un livre entier à la manière japonaise. Selon les mêmes procédés, Jacques Beltrand exécuta, entre 1900 et 1904, plusieurs estampes représentant des paysages parisiens ${ }^{19}$ (fig. 4). Sous leurs épreuves, il spécifiait soigneusement qu'elles avaient été « tirées à l'eau ».

16. Lyon, 7 septembre 1847 - Paris, 19 février 1904. La bibliographie sur cet artiste est très réduite. Nous renvoyons à la notice que nous lui consacrons dans le catalogue de l'exposition Japon-Paris-Bretagne (op. cit.), p. 16, suivie de la reproduction des rares estampes en couleurs de Tony Beltrand connues à ce jour. Tony Beltrand, comme Rivière, collectionnait les objets d'art japonais et les estampes.

17. Anatole Le Braz, Croquis de Bretagne et d'ailleurs. Bois originaux de Tony Beltrand, Paris, Louis Conard, 1903.

18. Paris, 22 juillet 1874 - Coucy-lès-Eppes, 2 décembre 1977.

19. La Rue Valette à Paris, 1901 ; La Place Saint-Michel, 1903. 

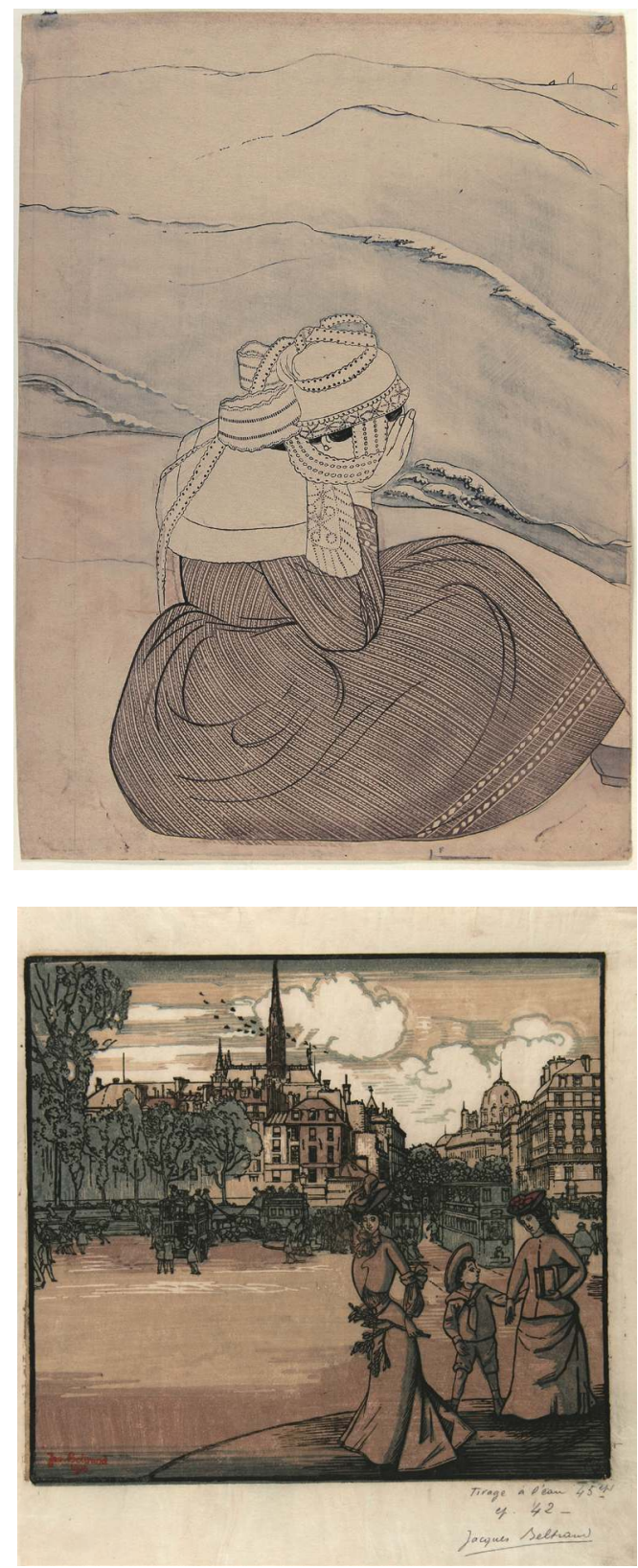

Fig. 3.

Tony Beltrand, Femme de Concarneau. Gravure sur bois imprimée en couleurs, à l'eau et gaufrage, 1897. H. $34 \mathrm{~cm}$; L. $24 \mathrm{~cm}$. Coll. particulière.

Fig. 4.

Jacques Beltrand, La Place Saint-Michel. Gravure sur bois imprimée en couleurs, à l'eau, 1903.

H. $23,5 \mathrm{~cm}$; L. $25,7 \mathrm{~cm}$. Coll. particulière. 


\section{Fig. 5.}

Maurice Denis, illustration gravée par Jacques Beltrand pour : André Suarès, Le Crépuscule sur la mer, Paris, 1938, p. 46. Gravure sur bois imprimée en couleurs.

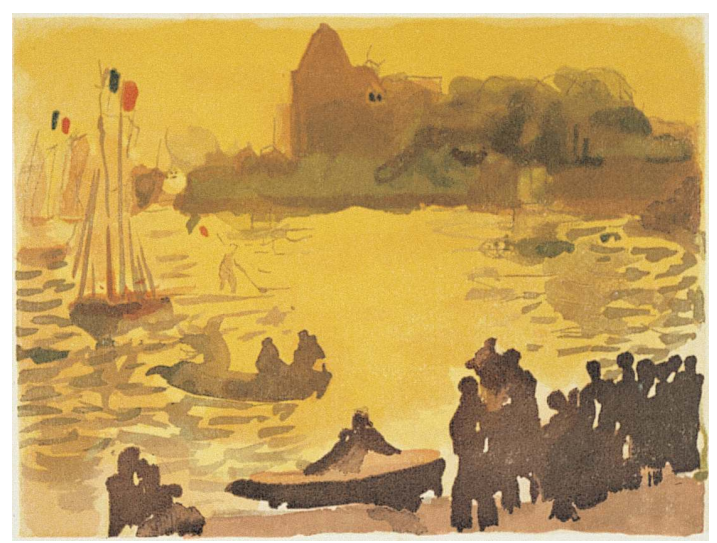
H. $11 \mathrm{~cm}$; L. $14,5 \mathrm{~cm}$. Coll. Musée départemental breton (Conseil général du Finistère), Quimper.

Ayant ainsi acquis une excellente maitrise de la gravure en couleurs, Jacques Beltrand fut ensuite le principal rénovateur de la gravure sur bois en camaïeu, c'est-à-dire en deux ou trois tons de la même couleur, procédé de tradition occidentale qui remontait au XVI ${ }^{\mathrm{e}}$ siècle, mais avait été à peu près abandonné après le XVII ${ }^{\mathrm{e}}$ siècle. Dans le domaine de l'édition, il fut durant plusieurs décennies le collaborateur du peintre Maurice Denis et réalisa avec lui plusieurs dizaines de livres ${ }^{20}$. La plupart sont illustrés de gravures sur bois gravées en couleurs par Beltrand d'après des dessins et des aquarelles de Denis (fig. 5). Ces illustrations montrent l'exceptionnelle virtuosité atteinte par Beltrand dans l'art de la gravure en couleurs. Précisons de nouveau que, si la technique de gravure sur plusieurs matrices imprimées au repérage était imitée du Japon, l'impression de ces livres et de leur illustration se fit à la presse et avec de l'encre grasse, conformément aux habitudes des ateliers français.

20. Entre autres : Verlaine, Sagesse, Paris, Vollard, 1911 ; Lacordaire, Vie de saint Dominique, Beltrand, 1919 ; André Suarès, Le Crépuscule sur la mer, Beltrand, 1933 ; Paul Claudel, L'Annonce faite à Marie, Paris, Blaizot, 1940 ; etc. 


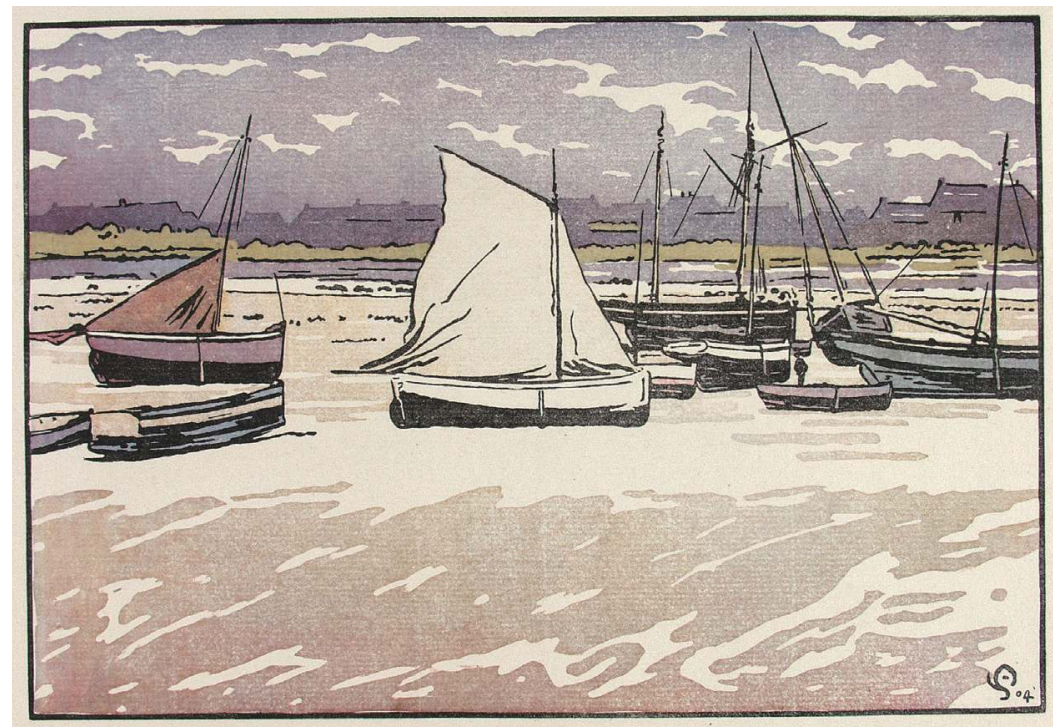

Fig. 6.

Amédée Joyau, Roscoff. Gros temps, marée basse.

Gravure sur bois imprimée en couleurs, à l'eau, 1904.

H. $20 \mathrm{~cm} ;$ L. $27,8 \mathrm{~cm}$. Coll. Musée départemental breton

(Conseil général du Finistère), Quimper.

\section{Deux graveurs sur bois en Bretagne, Amédée Joyau et Adolphe Beaufrère}

Une autre figure, tellement discrète qu'elle est à peu près complètement tombée dans l'oubli, est celle d'Amédée Joyau ${ }^{21}$. Comme Rivière, il apprit par lui-même les techniques japonaises de la gravure sur bois en couleurs.

21. Paris, 25 mai 1872 - Fontainebleau, 5 août 1913. Sarah Sauvin, qui a établi le catalogue du fonds Joyau de la Bibliothèque nationale de France, a rédigé la notice de ce graveur dans le catalogue de l'exposition Japon-Paris-Bretagne, op. cit., p. 30. Voir aussi : Atherton Curtis, Catalogue de l'auvre gravé d'Amédée Joyau, Paris, Prouté, 1938. 
Il les appliqua dans une série de scènes intimistes ainsi que dans quelques paysages parisiens ${ }^{22}$. Joyau fit aussi de nombreux paysages des provinces françaises de Normandie, de Bretagne ${ }^{23}$ (fig. 6) et de Vendée. Son œuvre nous mène aux toutes premières années $\mathrm{du} \mathrm{xx}^{\mathrm{e}}$ siècle. C'est aussi dans cette période que se situe la production de bois en couleurs d'Adolphe Beaufrère $^{24}$. Originaire de Bretagne, il choisit, après sa formation à Paris, de retourner vivre dans cette région où l'attirait le souvenir de Paul Gauguin. Il acquit surtout sa renommée comme graveur en taille-douce, mais il pratiqua aussi, à ses débuts, la gravure sur bois, technique à laquelle il s'initia auprès de Jacques Beltrand. En 1900 ou 1902 il grava et imprima à l'eau deux scènes de femmes à la toilette ${ }^{25}$. Leur technique d'exécution est japonaise, mais leur inspiration et leur composition le sont également. Elles trouvent en effet leur source dans des estampes d'Utamaro. Quant au buste assez patibulaire de la Vieille Bretonne (1904), il est, dans sa composition en plan rapproché, sans doute inspiré de certains portraits d'acteurs présents dans l'estampe japonaise.

\section{La transmission des techniques japonaises de l'estampe en couleurs, d'Urushibara à Geo-Fourrier}

Il est particulièrement intéressant d'étudier comment, dans le petit milieu des graveurs parisiens, se formèrent d'une génération à l'autre des filiations, forgées par l'amitié, et manifestées par la transmission d'outils et de savoirfaire. Presque tous ces graveurs se connaissaient. Plusieurs fréquentaient le cercle d'amateurs d'art oriental qui se réunissaient à Paris autour du joaillier et mécène Henri Vever (1854-1942). Depuis la mort de Siegfried Bing en 1905, Vever présidait la Société des amis de l'art japonais (fig. 7), dont firent partie un grand nombre d'artistes japonisants. L'arrivée dans ce cercle des japonisants de Paris du graveur et imprimeur Urushibara Yoshijirō

22. Anna, femme se recoiffant, 1899 ; Boulevard Malesherbes, Paris, 1898 ou 1899, etc.

23. Notamment, en 1903 et 1904, une suite consacrée au port de pêche de Roscoff, en Bretagne.

24. Quimperlé, 25 mars 1876 - Larmor-Plage, 16 février 1960. Voir : Daniel Morane, Adolphe Beaufrère, l'euvre gravé, Concarneau, galerie Gloux, 1981.

25. Femme au tub et La Toilette, vers 1902. 


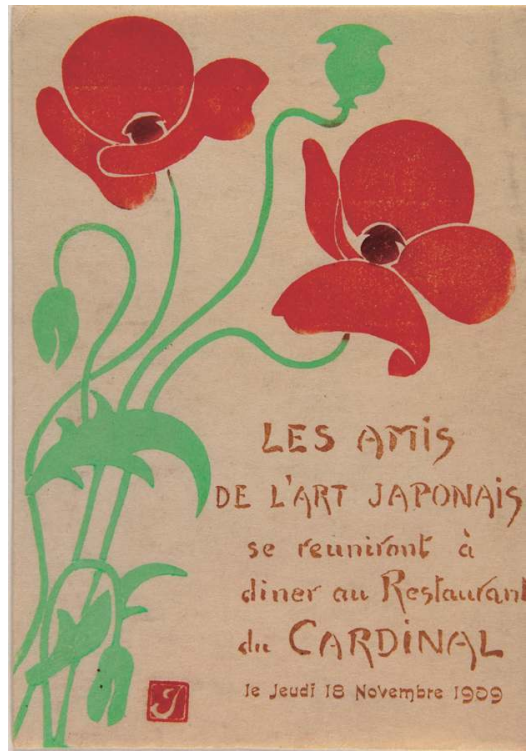

Fig. 7.

Prosper-Alphonse Isaac, invitation pour les

Amis de l'art japonais.

Gravure sur bois imprimée en couleurs, 1909.

H. $18,4 \mathrm{~cm}$; L. $12,3 \mathrm{~cm}$.

Coll. particulière.

Fig. 8.

Prosper-Alphonse Isaac, Cygne. Gravure sur bois imprimée en couleurs, à l'eau, vers 1910 . H. $12,5 \mathrm{~cm}$; L. $18,7 \mathrm{~cm}$. Coll. Musée départemental breton (Conseil général du Finistère), Quimper

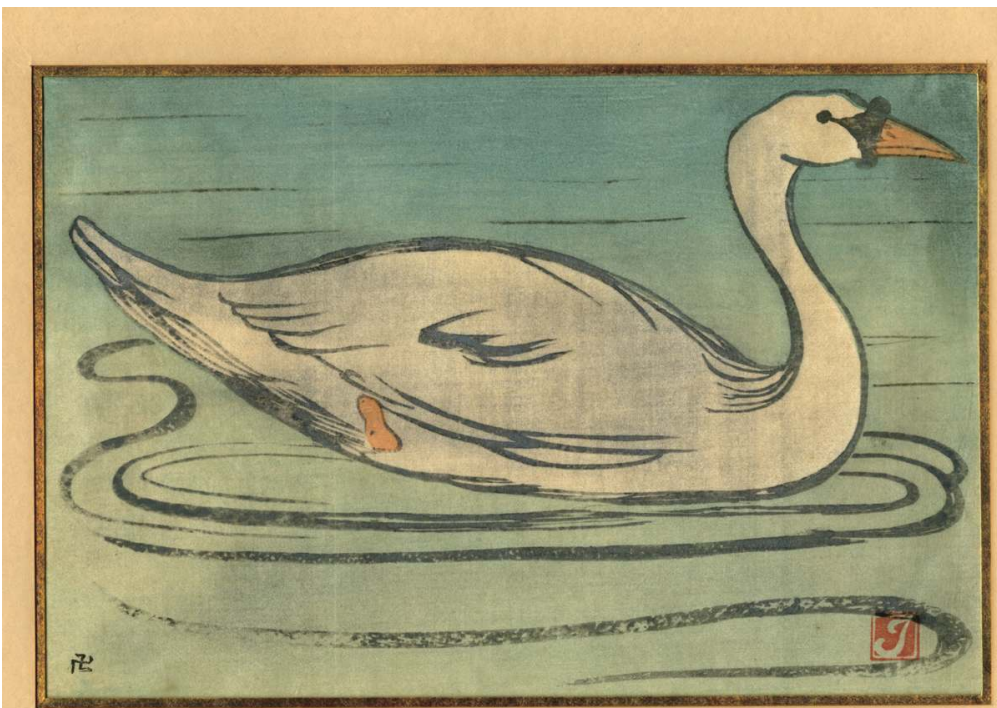


漆原由次郎 ${ }^{26}$, vers 1909-1910, contribua à étendre la pratique de la gravure sur bois en couleurs auprès de quelques graveurs et à provoquer ce que j'appellerais une seconde vague japoniste, qui s'épanouit à partir de la fin des années 1900 et durant tout l'entre-deux-guerres. En 1910, Urushibara avait été envoyé à Londres par son employeur, la maison d'édition Shimbi shoin 審美書院, afin d'exécuter des démonstrations d'impression selon les méthodes japonaises dans le cadre de l'Exposition anglo-japonaise qui se tenait dans la capitale britannique. Il y rencontra Henri Vever et ce fut sans doute à l'invitation de celui-ci qu'il se rendit ensuite en France. Une partie de son œuvre personnelle reflète d'ailleurs ses séjours à Paris ${ }^{27}$.

Dans cette ville, il se lia à Prosper-Alphonse Isaac ${ }^{28}$. Celui-ci pratiquait la gravure sur bois en couleurs avant de connaître Urushibara, mais de manière encore relativement rudimentaire, notamment pour la gravure d'invitations pour les Amis de l'art japonais. Auprès d'Urushibara, Isaac se perfectionna dans les techniques d'encrage et d'impression au point d'atteindre une véritable expertise dont il put ensuite faire profiter d'autres graveurs et dessinateurs. Isaac et Urushibara exécutèrent ensemble plusieurs estampes qui portent leurs deux monogrammes. Par exemple, sur une épreuve conservée à la Bibliothèque nationale de France de la Carcasse de bateau à Cancale (1912) d'Isaac, on distingue à droite le monogramme d'artiste de ce dernier ; à gauche, son emblème en tant qu'imprimeur - un sauvastika - et le cachet d'Urushibara.

Isaac employa sa connaissance des procédés japonais pour graver ses propres compositions (fig. 8), mais il les mit aussi au service d'autres artistes. Il eut ainsi un véritable rôle de propagateur. C'est à lui que les graveurs français doivent le premier exposé technique, exact et précis, des procédés japonais de gravure et d'impression. Publiée dans la revue Art et Décoration en mai $1913^{29}$, cette présentation était illustrée de photographies montrant

26. Tokyo, 12 mars 1889 - Tokyo, 6 juin 1953.

27. Urushibara Yoshijirō, Notre-Dame de Paris. Gravure sur bois imprimée en couleurs, à l'eau, après 1913 .

28. Calais, 1858 - Paris, 22 juin 1924. Voir : Émilie Vabre, "Prosper-Alphonse Isaac, graveur sur bois à la manière japonaise ", Nouvelles de l'estampe, $\mathrm{n}^{\circ} 237$, hiver 2011 2012, p. 4-21.

29. Prosper-Alphonse Isaac, "La gravure sur bois à la manière japonaise ", Art et Décoration, mai 1913, p. 155-162. 
les différentes phases de l'élaboration d'une estampe ainsi que les outils nécessaires à son exécution.

En 1905, alors qu'il poursuivait chez Vever une carrière de dessinateur de bijoux, Jules Chadel $^{30}$ eut à son tour la révélation : "Quand je connus les grands Japonais », racontait-il, « il me sembla que j'avais à recommencer toute mon éducation ${ }^{31}$ ». Chadel pratiquait principalement l'aquarelle et le lavis, dans un style graphique influencé par le dessin japonais. Isaac et Urushibara lui enseignèrent l'art de traduire ses lavis dans le bois et de les reproduire en impression sans rien leur ôter de leur fluidité. Dans ses estampes, on admire la virtuosité atteinte pour rendre en xylographie la délicatesse et la transparence de l'aquarelle, au point qu'au premier regard, on pourrait prendre ces épreuves de gravures sur bois pour des dessins originaux ${ }^{32}$ (fig. 9). La collaboration entre Chadel et Urushibara a été récemment étudiée, notamment à la lumière de documents japonais, par Mayumi Nakamura, dont je salue le travail ${ }^{33}$. Cette collaboration entre le Japonais et le Français s'est spécialement manifestée dans le domaine du livre de luxe : Urushibara imprima les bois, gravés par Chadel, de trois ouvrages commandés par des sociétés de bibliophilie ${ }^{34}$. La réalisation de ces ouvrages montre un autre exemple de filiation et d'apprentissage entre graveurs. Chadel se fit en effet assister pour leur gravure par son élève Germaine de $\operatorname{Coster}^{35}$. Les outils de gravure de Germaine de Coster se trouvent aujourd'hui à la Bibliothèque nationale de France. Certains d'entre eux lui avaient été offerts par Urushibara.

30. Clermont-Ferrand, 10 juin 1870 - Paris, 16 décembre 1942. Voir notre notice dans Japon-Paris-Bretagne, op. cit., p. 21.

31. Propos de Chadel cité dans : Léon Deshairs, "Les dessins de Jules Chadel ", Art et Décoration, novembre 1922, p. 133.

32. Voir par exemple sa Pie mouillée, 1920 ou ses paysages gravés du port de Douarnenez, en Bretagne (1922 et 1930).

33. Mayumi Nakamura, Transmission de la technique de la gravure sur bois de la fin du $X I X^{e}$ au début du XX $X^{e}$ siècle : la collaboration entre Jules Chadel et Urushibara Yoshijirō, mémoire de master 2 d'histoire de la création artistique occidentale, université de Paris IVSorbonne, juin 2013.

34. Quelques fables de La Fontaine, Paris, Les cent Bibliophiles, 1927 ; Maurice de Guérin, Le Centaure et la Bacchante, Paris, Le livre contemporain, 1931 ; Évangile selon saint Luc, Châtenay-Malabry, Pro Amicis, 1932.

35. Paris, $1^{\text {er }}$ septembre 1895 - Paris, décembre 1992. 


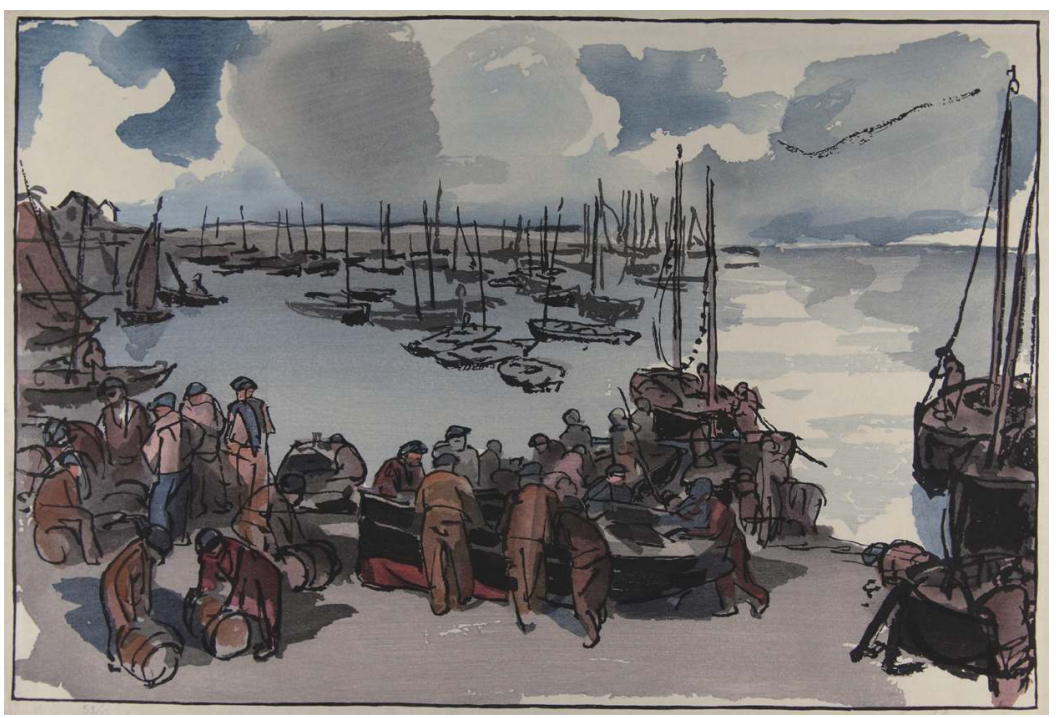

Fig. 9. Jules Chadel, Douarnenez.

Gravure sur bois imprimée en couleurs, à l'eau, 1930.

H. 29,4 cm ; L. 44 cm. Coll. Musée départemental breton

(Conseil général du Finistère), Quimper.

Un autre processus de transmission relie Isaac au dessinateur et graveur Geo-Fourrier ${ }^{36}$ (1898-1966). Passionné très jeune par le Japon, il fréquentait les musées et y recopiait dans des carnets les objets et motifs japonais qu'il admirait. En 1920 et 1921, il se forma auprès d'Isaac aux techniques japonaises d'impression en couleurs auxquelles il consacra plus tard une étude, demeurée inédite et reliée par lui-même, selon la méthode utilisée pour les livres japonais ${ }^{37}$. Il se fixa à Quimper au milieu des années 1920 et l'essentiel de son ouvre est d'inspiration bretonne. Il comporte notamment un petit nombre de bois gravés et imprimés par lui-même, à l'eau,

36. Lyon, 16 juin 1898 - Quimper, 8 avril 1966. Voir sur cet artiste notre introduction à : Geo-Fourrier, Les Bigoudens, Paris, Asia, 2008.

37. Geo-Fourrier, La technique de l'estampe japonaise, 1946 (tapuscrit inédit). 
en 1925 et 1926. Mais par la suite, Geo-Fourrier s'inscrivit complètement, pour la gravure et l'impression de ses bois en couleurs, dans le processus japonais d'exécution des estampes, en en confiant l'exécution à des graveurs et imprimeurs professionnels japonais. C'est-à-dire qu'il fit graver et imprimer au Japon, d'après des aquarelles qu'il y expédiait, plusieurs grandes compositions à thème breton, reportées dans le bois et tirées à Tokyo par l'éditeur Takamizawa Mokuhansha 高見澤木版社, spécialisé dans l'édition et la réédition d'estampes anciennes.

Pour ses Costumes de fêtes, estampe gravée en 1937 et imprimée en couleurs l'année suivante (fig. 10), Geo-Fourrier souhaitait qu'elle soit imprimée sur fond micassé, spécialité que seul un imprimeur japonais pouvait réaliser. Inspirée par les costumes des environs de Quimper, cette estampe

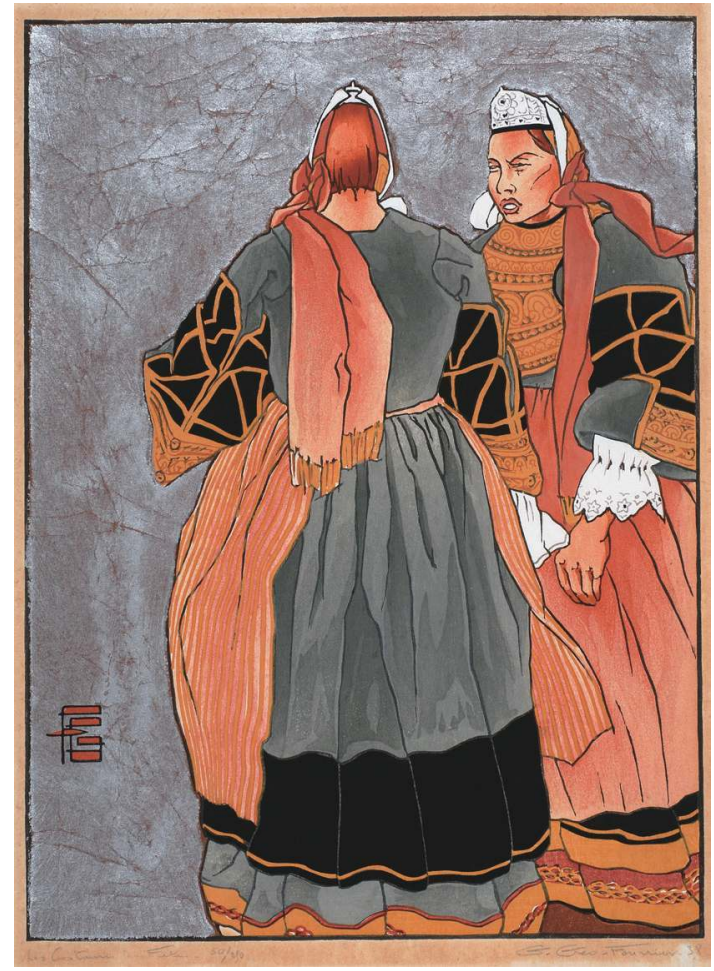

Fig. 10.

Geo-Fourrier, Les costumes de fêtes. Gravure sur bois imprimée en couleurs, à l'eau, par l'atelier Takamizawa Mokuhansha (Tokyo), 1938.

H. $35,1 \mathrm{~cm}$; L. $25,6 \mathrm{~cm}$. Coll. Musée départemental breton (Conseil général du Finistère), Quimper. C ASIA Éditeur, 2014 pour l'œuvre de Geo-Fourrier (102 bd. Kerllerman, 75013 Paris, asia-editeur.com). 
fut gravée et imprimée à Tokyo, comme l'atteste le cachet qui figure au revers des épreuves. Ce recours à un atelier professionnel japonais est un exemple dont je ne connais pas d'équivalent à cette époque de la part d'un artiste vivant en France. Il permit à Geo-Fourrier d'obtenir des estampes remarquablement fidèles à son dessin, comme le montre la comparaison entre la gouache originale du Brûleur de goémon en Bretagne, de 1935, acquise depuis par le Musée départemental breton de Quimper, et l'estampe exécutée à Tokyo l'année suivante.

Geo-Fourrier fut aussi un illustrateur, mettant à profit sa maîtrise de la gravure en couleurs. Dans cette partie de sa production cependant, selon la pratique que j'ai déjà soulignée à plusieurs reprises, les bois en couleurs furent imprimés à la presse et à l'encre grasse et non selon la méthode japonaise. Ce fut par exemple le cas pour ses illustrations des Hommes nouveaux, roman de Claude Farrère dont l'action se déroule en Afrique du Nord ${ }^{38}$.

\section{La gravure en couleurs au-delà du japonisme, de Daragnès à Clairin}

Malgré la prépondérance des techniques occidentales dans le livre illustré, les études que je mène à la fois sur l'histoire de l'estampe et sur celle du livre français me convainquent que la vogue du bois imprimé en couleurs dans l'illustration, au cours des années 1930 à 1950, fut la suite logique de la découverte des techniques japonaises par les graveurs français à la fin du $\mathrm{XIX}^{\mathrm{e}}$ siècle. Là encore, les filiations sont intéressantes à relever. J'en donnerai un dernier exemple : Jean-Gabriel Daragnès ${ }^{39}$, figure majeure de l'édition bibliophilique française du $x^{e}$ siècle, commença sa carrière à la fin des années 1910, c'est-à-dire à l'époque où s'affirmaient l'œuvre d'Isaac ou celle de Chadel. Dans les années 1930, il fut avec Jacques Beltrand le principal représentant français de l'art de la gravure sur bois en couleurs appliquée au livre ${ }^{40}$.

38. Claude Farrère, Les Hommes nouveaux, Paris, Horizons de France, 1928.

39. Bordeaux, 2 avril 1886 - Neuilly-sur-Seine, 25 juillet 1950.

40. Parmi les livres qu'il illustra de gravures sur bois imprimées en couleurs, on citera seulement : Le Roman de Tristan et d'Iseult, 1928; La Chanson de Roland, 1932 ; Gustave 
Au lendemain de la Seconde Guerre mondiale, Daragnès initia à la gravure sur bois en couleurs le peintre Pierre-Eugène Clairin ${ }^{41}$. Clairin à son tour illustra de bois en couleurs plusieurs beaux livres, sans cependant recourir à l'impression "à la japonaise " ${ }^{42}$. Adepte de l'estampe en couleurs, il fut également un spécialiste de la lithographie. Il entretint des relations assez étroites avec le Japon et se lia au grand poète et romancier Kawabata Yasunari, qu'il évoque dans sa lithographie Le poète Kawabata sous la neige (1970). Kawabata préfaça le catalogue de l'exposition de Clairin, à la galerie Marsuya de Tokyo en 1970.

J'ai retrouvé des lettres de Clairin écrites à son retour du Japon. Il y avait été invité par l'atelier de gravure Fuji bijutsu 富士美術 pour y enseigner la technique de la lithographie. Un peu comme Urushibara qui à Paris, vers 1910, enseignait l'impression en couleurs des gravures sur bois. Voici ce qu'écrivait Clairin au critique et historien de la gravure Claude RogerMarx, en février 1970 : "Invité par une société montant une imprimerie lithographique j'ai formé deux jeunes pressiers et en somme débuté la litho au Japon où elle est tout-à-fait débutante ${ }^{43}$ ". Cette citation conclura notre propos tout entier consacré à la " leçon japonaise " suivie par des graveurs français. À travers l'exemple de Clairin s'est manifestée en somme, et à l'échelle certes bien plus réduite, une petite "leçon française " auprès des graveurs japonais.

Flaubert, La Tentation de saint Antoine, 1942.

41. Cambrai, 14 mars 1897 - Thorigné-en-Charcy, 7 juillet 1980.

42. Henri de Montherlant, Une Aventure au Sahara, éditions des XXX Lyonnais, 1951 ; Paul-Jean Toulet, La Jeune Fille verte, Paris, Le Livre contemporain, 1953.

43. Clairin à Claude Roger-Marx, 9 février 1970. Paris, INHA, fonds Claude RogerMarx, boîte $n^{\circ} 35$. 


\section{Une leçon japonaise : la gravure sur bois en couleurs en France selon les procédés japonais (1889-1939)}

1889 ～ 1939 年のフランスの木版表現にみられる

浮世絵の影響、そして出版

フィリップ・ル・ストウム

The Colour Woodcut in France

Using Japanese Techniques (1889-1939)

Philippe Le STUM

\section{\ots-clés :}

estampe, gravure sur bois, japonisme.

L'auteur: Conservateur, directeur du Musée breton (Quimper) et docteur en histoire de l'art, Philippe Le Stum a étudié la diffusion des procédés japonais de gravure dans des expositions et ouvrages : Henri Rivière, 2002 ; La gravure sur bois en Bretagne, 2005 ; Japon-Paris-Bretagne, la gravure sur bois en couleurs, 2012, etc.

Résumé : L’utilisation des procédés japonais de production d'estampes par quelques graveurs français s'inscrivit dans le double mouvement de renouveau de la gravure sur bois originale et d'avènement de la couleur dans la gravure occidentale. Le japonisme s'y manifesta par l'expérimentation de techniques xylographiques et de procédés d'impression : gravure d'un bloc de bois par couleur, pigments de l'aquarelle, tirage " à l'eau ". Les précurseurs de ce mouvement furent Henri Rivière et Auguste Lepère à la fin des années 1880. Suivirent les productions d'Amédée Joyau, Tony et Jacques Beltrand ou Adolphe Beaufrère. Au début du $\mathrm{xx}^{\mathrm{e}}$ siècle, le japonisme xylographique français se prolongea dans l'œuvre de Prosper-Alphonse Isaac et Jules Chadel puis, jusque dans les années 1920 et 1930, dans celle de Geo-Fourrier. 
〉キーワード

版画、木版画、ジャポニスム

著者

ブルターニュ地方美術館（カンペール）館長。 美術史で博士号を取得。日本の版画技法の伝 播に関する展覧会の企画や著作の執筆を行う。

\section{要旨}

フランスの版画家の中には日本の版画技法を 用いた者がいたが、それはオリジナル木版画 の復権と西洋の版画における色彩の出現とい うふたつの動向の中に位置づけられる。色彩 を用いた木版、水彩顔料の使用、ぼかしなど、 木版画の技法を実験的に用いたジャポニスム もこの流れの中に現れる。先駆者は 1880 年 代末に活躍したアンリ・リヴィエールとオー ギュスト・ルペールである。彼らの後にはア メデ・ジョワイヨ、ベルトラン親子、アドルフ・ ボーフレールが続いた。20 世紀初めにはフラ ンスの木版画におけるジャポニスムはプロス ペールニアルフォンソ・イサックやジュール． シャデルへと、さらに 1920 年代および 30 年 代にはジェオ・フリエに継承された。
Ceywords: Print, Woodcut, Japonism.

The Author: Curator, director of the $\mathrm{Mu}$ sée Breton (Quimper) and a Doctor of Art History, Philippe Le Stum has studied the dissemination of Japanese printmaking techniques in a variety of exhibitions and books including Henri Rivière, 2002; La gravure sur bois en Bretagne, 2005; and Japon-Paris-Bretagne, la gravure sur bois en couleurs, 2012.

\begin{abstract}
The use of Japanese printmaking techniques by a handful of French engravers was part of a dual process involving the revival of the original woodcut and the advent of colour in Western printmaking. The influence of Japonism was apparent in the experimentation with xylography techniques and printing methods: engraving of one woodblock per colour, use of watercolour pigments, and water-colour printing. Henri Rivière and Auguste Lepère were pioneers in this movement in the late 1880s, followed by the work of Amédée Joyau, Tony and Jacques Beltrand, and Adolphe Beaufrère. In the early twentieth century French Japanese-style woodcuts continued in the work of ProsperAlphonse Isaac and Jules Chadel then, during the 1920s and 1930s, in the work of Geo-Fourrier.
\end{abstract}

